\title{
EYA1 nonsense mutation in a Japanese branchio-oto-renal syndrome family
}

\begin{abstract}
Advances in molecular genetics have recently revealed that mutations in the EYAl gene are responsible for branchio-oto-renal (BOR) syndrome in European and other populations. This is the first report confirming that an EYAl gene mutation is also disease-causing in an Asian population. We have described one Japanese BOR syndrome family showing a novel mutation in exon 7 of the EYAl gene. There was extensive variation of clinical phenotypes within this family. When the physician is confronted with a BOR family showing a wide variation in clinical expression, molecular genetic testing helps to achieve accurate diagnosis.
\end{abstract}

Key words Branchio-oto-renal (BOR) syndrome $E Y A 1$. Mutation $\cdot$ Japanese $\cdot$ Hearing impairment

\section{Introduction}

Branchio-oto-renal (BOR) syndrome is an autosomal dominant inherited disorder, associated with: (1) branchial cleft, fistulas or, cysts; (2) various ear anomalies, including malformed pinnae, preauricular pits or sinuses, occicular anomalies, or inner ear anomalies; and (3) renal abnormalities (McKusick 1994). Prevalence of the BOR syndrome has been estimated as 1:40,000, and therefore may account for as much as $2 \%-3 \%$ of profound hearing loss in children (Fraser et al. 1980).

Recent advances in molecular genetics have revealed that the gene responsible for BOR syndrome is localized in 8q (Kumar et al. 1992; Smith et al. 1992), and that there were various mutations in the EYAl gene, the human ho-

S. Usami $(\square) \cdot$ S. Abe $\cdot$ H. Shinkawa

Department of Otorhinolaryngology, Hirosaki University School of Medicine, 5 Zaifu-cho, Hirosaki 036-8562, Japan

Tel. +81-172-39-5099; Fax +81-172-39-5100

e-mail: usami@cc.hirosaki-u.ac.jp

K. Deffenbacher · S. Kumar · W.J. Kimberling Department of Genetics, Boys Town National Research Hospital, Omaha, USA mologue of the Drosophila eyes absent gene, in Northern European and other BOR patients (Abdelhak et al. 1997a; Abdelhak et al. 1997b; Kumar et al. 1997/98; Kumar et al. 1998a; Kumar et al. 1998b).

In the present study, both detailed clinical evaluation and mutation analysis were carried out in a Japanese BOR syndrome family and it was confirmed that the EYA1 gene is also disease-causing in an Asian population.

\section{Case report}

The pedigree of the present family is shown in Fig. 1. Two brothers (numbers 2 and 3 in the pedigree), out of three siblings, were referred to the Otorhinolaryngology Department, Hirosaki University Hospital, Hirosaki, Japan, because of bilateral hearing loss. Their father (number 4 in the pedigree) also had hearing loss, but their mother and sister were unaffected. The paternal grandmother was reported to have preauricular pits. The children's parents were not consanguineous. All three affected family members had mild "cup-shaped" pinnae and bilateral preauricular fistulae. The younger brother had left cervical branchial fistula. The affected individuals were clinically evaluated with puretone audiometry, computed tomographic (CT) scan, ultrasound scan of the kidneys, and intravenous urography. Written informed consent was obtained from all subjects prior to performance of the study.

Pure-tone audiometry showed conductive hearing loss of $20-50 \mathrm{~dB}$ in the elder brother (Fig. 2A). The father and younger brother had bilateral mixed hearing loss ranging from $20 \mathrm{~dB}$ to $80 \mathrm{~dB}$ (Fig. 2B, C). Progression of hearing loss was not evident in any of these three individuals. CT scans revealed bilateral hypoplasia of the cochlea, bilateral hypoplasia of the lateral and posterior semicircular canal, bilateral abnormal ossicular chain (laterally shifted malleus), and soft mass density in the epitympanic and mastoid cavity (elder brother, right; younger brother, right; father, bilateral) (Fig. 3A). Congenital cholesteatoma was confirmed by exploratory tympanotomy in the two children. Bilateral 
Fig. 1. Pedigree of the branchiooto-renal (BOR) syndrome family. Filled symbols represent affected family members. All family members are Japanese and reside in Aomori Prefecture in northern Japan. The clinical manifestations are presented in the inset
Fig. 2A-C A collection of audiograms from the patients (A elder brother, $\mathbf{B}$ father, $\mathbf{C}$ younger brother) which show conductive (A) and mixed $(\mathbf{B}, \mathbf{C})$ hearing loss

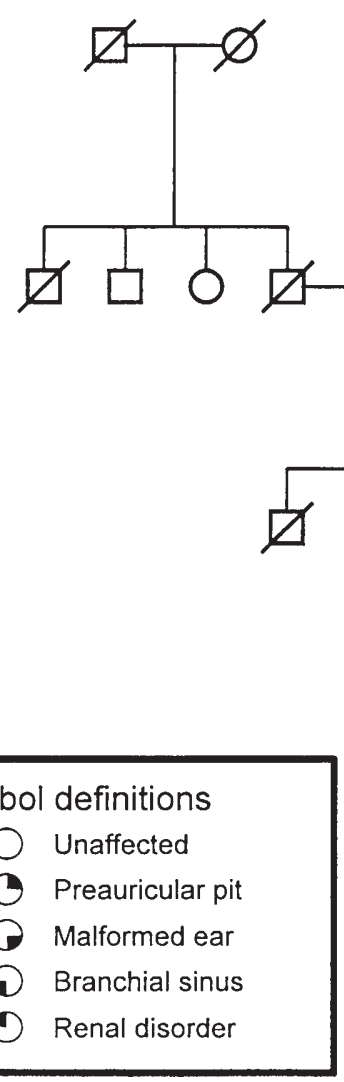

A

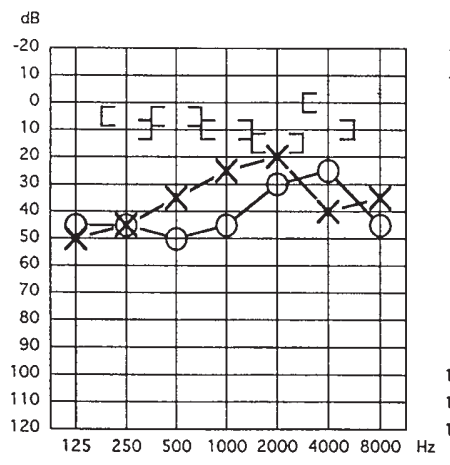

B

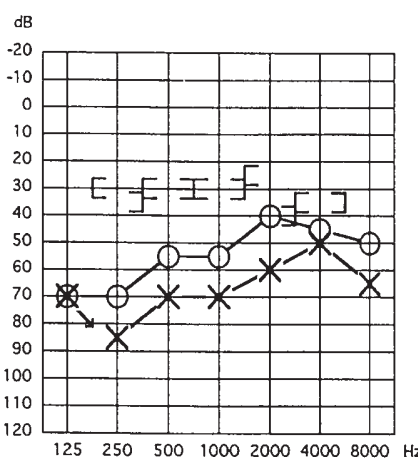

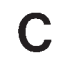

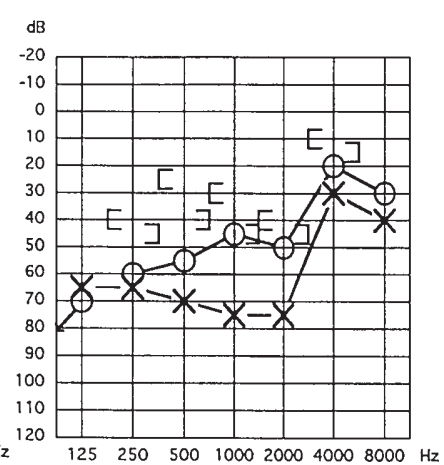

renal hypoplasia (right kidney length, $81.5 \mathrm{~mm}$; left kidney length, $75.4 \mathrm{~mm}$, measured by ultrasonography) was identified in the father (Fig. 3B). However, there were no renal cysts or any structural anomalies of the renal collecting system diagnosed by intravenous urography. The two affected children had normal appearance of the kidneys.

To detect EYA1 mutations, two methods were used in this study: heteroduplex analysis and direct sequencing, as described in detail elsewhere (Kumar et al. 1998a). In brief, total genomic DNA was extracted from the blood, and each exon of the EYAl gene was amplified, using intronic primers. For heteroduplex analysis, the polymerase chain reaction (PCR) products were heated to $95^{\circ} \mathrm{C}$ in the presence of ethylenediaminetetraacetic acid (EDTA) for $3 \mathrm{~min}$ and then were slowly cooled to $25^{\circ} \mathrm{C}$ over $45 \mathrm{~min}$. The reannealed reaction products were electrophoresed on a mutation detection enhancement (MDE) gel (FMC Bioproducts, Rockland, Maine, USA). Homoduplex and heteroduplex were visualized on a UV transilluminator, after staining in ethidium bromide. The PCR products were purified and sequenced by means of an ABI Prism BigDye Terminator Cycle Sequencing Ready Reaction Kit and an ABI sequencer 377 (Perkin Elmer, Foster City, CA, USA).

Heteroduplex analysis of exon 7 of the EYAl gene showed an altered banding pattern in affected family members as compared with unaffected members (Fig. 4A). Direct sequencing revealed a nonsense mutation: Y193stop a C-to-G transition at the residue of the nucleotide of 579 


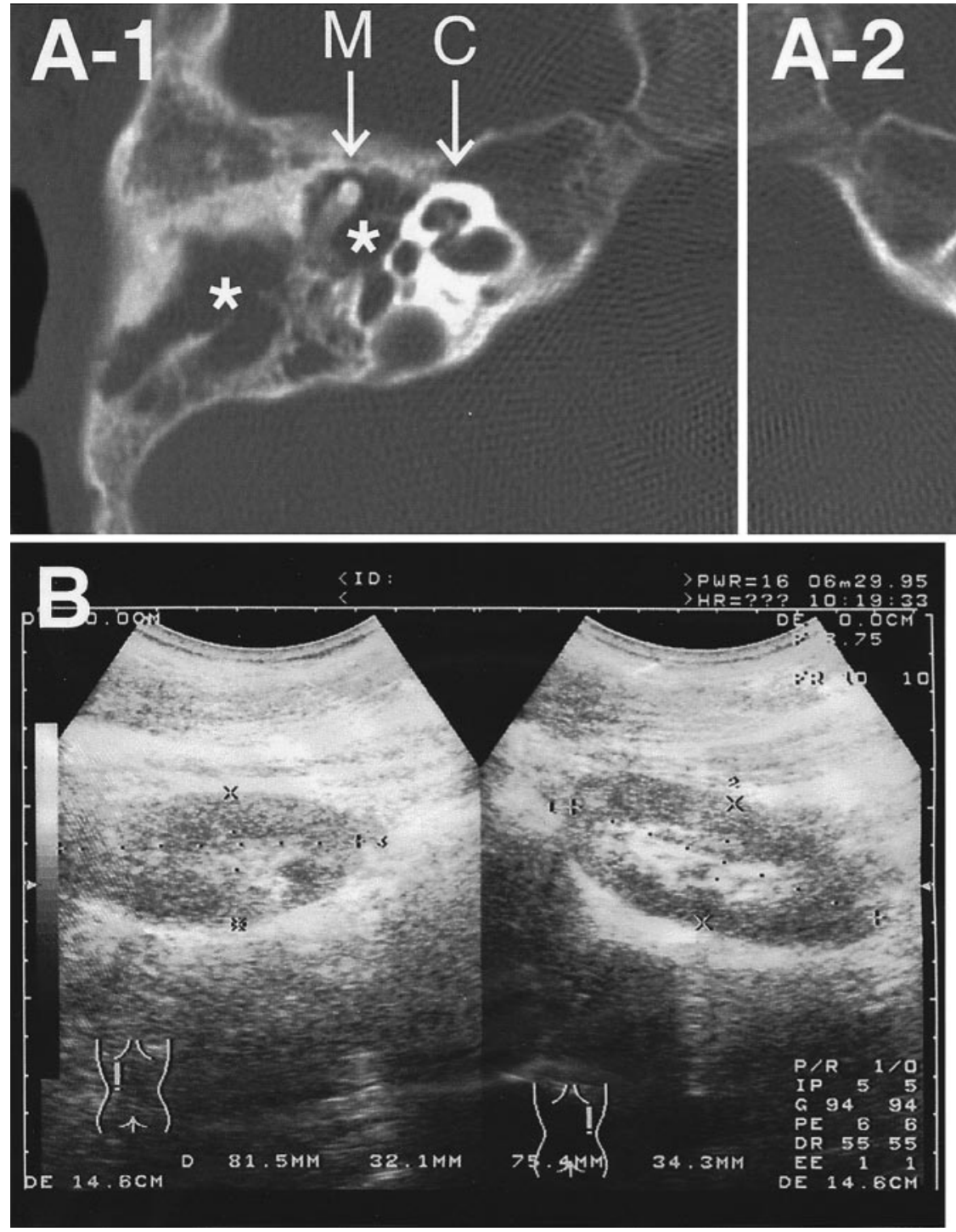

Fig. 3. A-1, A-2 Temporal bone computed tomography $(\mathrm{CT})$ scan in the elder brother, showing bilateral cochlear hypoplasia, indicated by $C$, and abnormal occicular chain (laterally shifted malleus), indicated by $M$. Soft mass density, indicated by asterisks, is found in the right epitympanic and mastoid cavity. B Ultrasonogram showing hypoplasia of bilateral kidneys in the father
(C579G) of the EYA1 genomic sequence. This nonsense mutation converts a tyrosine residue (TAC) at codon 193 to a stop codon (TAG) (Fig. 4B).

\section{Discussion}

This report clinically and genetically describes a wellcharacterized BOR syndrome in a Japanese family.

Twenty mutations have so far been described in the EYA1 gene (encompassing 16 exons) in BO/BOR families (Abdelhak et al. 1997a; Abdelhak et al. 1997b; Kumar et al. 1997/98; Kumar et al. 1998a; Kumar et al. 1998b; Vincent et al. 1997).

The present report adds a novel nonsense mutation in the $E Y A 1$ gene, and elucidates that mutations in the $E Y A 1$ gene are found across different ethnic groups. A single-base substitution introduces a stop codon in exon 7 in the EYAI gene, which results in an immature polypeptide. The segregation of this nonsense mutation with phenotypic expres- sion indicated that the allelic defect of the EYA1 gene may have led to the various phenotypes found in the present family.

The most characteristic clinical feature of BOR syndrome is its variable phenotypic expression. Although patients without renal abnormalities were sometimes considered to have $\mathrm{BO}$ syndrome, recent reports have categorized them as part of a single BOR condition (Allanson 1995). In BOR syndrome, hearing loss is the most prevalent symptom (89\%), followed by preauricular pits $(77 \%)$, branchial cleft fistulas (63\%), and renal anomalies (13\%) (Fraser et al. 1980). However, previous reports regarding renal anomalies have probably underestimated them because they were usually minor and asymptomatic (see Allanson 1995 for review).

A recent paper describing phenotypic manifestations associated with BOR syndrome also demonstrated a high prevalence $(67 \%)$ of renal malformation, assessed by ultrasonography and excretory urography (Chen et al. 1995). The present family showed variability: all had hear- 


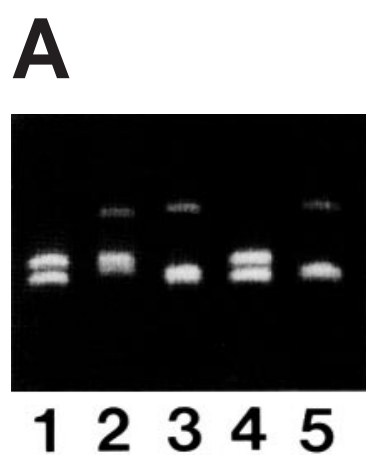

B

\section{CAGTACGCACAGTATT}
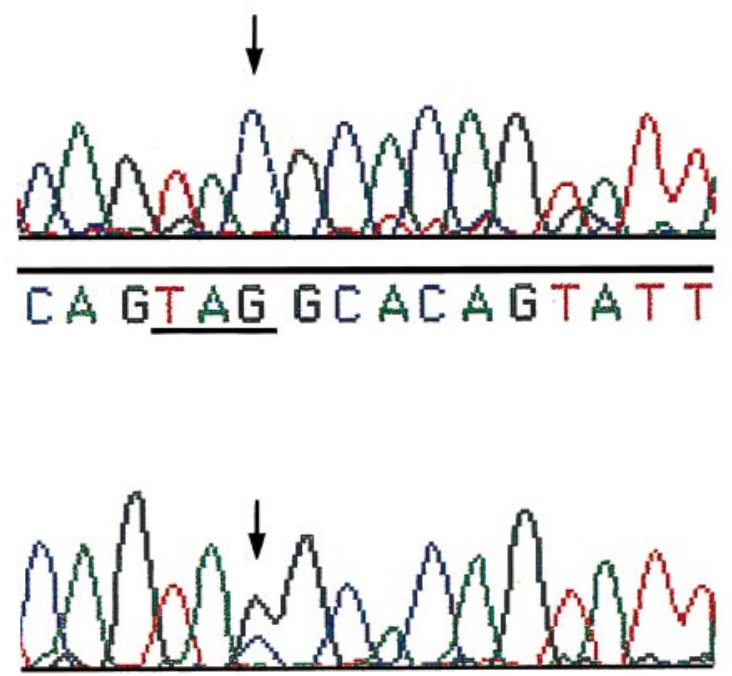

Fig. 4. A Heteroduplex analysis of exon 7 of the $E Y A 1$ gene showed an altered banding pattern in affected family members (lanes 2, 3, 5) as compared with unaffected members (lanes 1, 4). B Identification of mutation in exon 7 of the EYA1 gene. Electrophoretograms of a normal (upper) and a mutant (lower) allele. Heterozygote sequence showing C-to-G transition at the residue of the nucleotide of 579. This nonsense mutation converts a tyrosine residue (TAC) at codon 193 to a stop codon (TAG)

ing loss, one out of three had unilateral branchial cleft fistulas, and one had renal anomaly. According to the BO/BOR classification, the father's condition corresponded with BOR syndrome and the two affected childrens' with BO syndrome. It is noteworthy that the same mutation in the responsible gene could cause various conditions, although other reported mutations in the EYA1 gene may also lead to a wide range of phenotypic expression (Kumar et al. 1998b; Vincent et al. 1997). Recently, genetic heterogeneity associated with branchial and hearing anomalies has also been reported (Kumar et al. 1998b; Stratakis et al. 1998).

It has been reported that hearing loss may be either conductive $(30 \%)$, sensorineural $(20 \%)$, or mixed $(50 \%)$. The present study showed mixed as well as conductive hearing loss, and no specific genotypic association with hearing loss could be established in this syndrome.

Recent advances in computed tomography have revealed a high incidence of association with inner and ossicle malformations in BOR families (Chen et al. 1995). All of our patients had the characteristic features of BOR syndrome: hypoplasia of the cochlea, hypoplasia of the lateral and posterior semicircular canal, and abnormal ossicular chain (laterally shifted malleus) (Fig. 3). Two patients in the present family had congenital cholesteatoma. Although there have been few reports regarding the association of congenital cholesteatoma with BOR syndrome (Lipkin et al. 1986; Kusano et al. 1997), careful attention should be paidas to whether the individual patient has this disease, which requires surgery.

In conclusion, this report confirmed that an EYAl gene mutation is disease-causing in an Asian population, and the variation of clinical phenotypes shown within this family indicated that the single mutation could cause a broad phenotype, ranging from $\mathrm{BO}$ to $\mathrm{BOR}$. The reason for the various phenotypic expressions caused by only one mutation is unclear. It is possible that mutations in other deafness genes could also contribute to such various phenotypes. Molecular diagnosis of BOR syndrome, as well as genetic counseling, should be facilitated for accurate diagnosis.

Acknowledgments We thank the family that participated in the present project. We would also like to thank A.C. Apple-Mathews and Y. Kon for help in preparing the manuscript.

This study was supported by a Health Sciences Research Grant (Research on Eye and Ear Science, Immunology, Allergy and Organ Transplantation) from the Ministry of Health and Welfare of Japan (S.U.), and by NIH-NIDCD PO1 DC01813 (S.K.; W.J.K.)

\section{References}

Abdelhak S, Kalatzis V, Heilig R, Compain S, Samson, D, Vincent C, Levi-Acobas F, Cruaud C, Le Merrer M, Mathieu M, Konig R, Vigneron J, Weissenbach J, Petit C, Weil D (1997a) Clustering of mutations responsible for branchio-oto-renal (BOR) syndrome in the eyes absent homologous region (eyaHR) of EYA1. Hum Mol Genet 6:2247-2255

Abdelhak S, Kalatzis V, Heilig R, Compain S, Samson, D, Vincent C, Weil D, Cruaud C, Sahly I, Leibovici M, Bitner-Glindzicz M, Francis M, Lacombe D, Vigneron J, Charachon R, Boven K, Bedbeder P, Regemorter NV, Weissenbach J, Petit C (1997b) A human homologue of the Drosophila eyes absent gene underlies branchiooto-renal (BOR) syndrome and identifies a novel gene family. Nat Genet 15:157-164

Allanson J (1995) Genetic hearing loss associated with external ear abnormalities: branchio-oto-renal (BOR) syndrome (branchiooto-syndrome, ear-pit hearing loss syndrome). In: Gorlin RJ, Toriello HV, Cohen MM (eds) Hereditary hearing loss and its syndromes. Oxford University Press, New York, pp 76-80

Chen A, Francis M, Ni L, Cremers CWRJ, Kimberling WJ, Sato Y, Phelps PD, Bellman SC, Wagner MJ, Pembrey M, Smith RJH (1995) Phenotypic manifestations of branchio-oto-renal syndrome. Am J Med Genet 58:365-370 
Fraser FC, Sproule JR, Halal F (1980) Frequency of the branchio-otorenal (BOR) syndrome in children with profound hearing loss. Am J Med Genet 7:341-349

Kumar S, Kimberling WJ, Kenyon JB, Smith RJ, Marres HA, Cremers CW (1992) Autosomal dominant branchio-oto-renal syndrome localization of a disease gene to chromosome $8 \mathrm{q}$ by linkage in a Dutch family. Hum Mol Genet 1:491-495

Kumar S, Deffenbacher K, Cremers CWRJ, Van Camp G, Kimberling WJ (1997/98) Branchio-oto-renal syndrome: identification of novel mutations, molecular characterization, mutation distribution, and prospects for genetic testing. Genet Testing 1:243-251

Kumar S, Kimberling WJ, Weston MD, Schaefer BG, Berg MA, Marrer HA, Cremers CW (1998a) Identification of three novel mutations in human EYA1 protein associated with branchio-oto-rena syndrome. Hum Mutat 11:443-449

Kumar S, Marres HA, Cremers CW, Kimberling WJ (1998b) Autosomal-dominant branchio-otic (BO) syndrome is not allelic to the branchio-oto-renal (BOR) gene at 8q13. Am J Med Genet 76:395-401
Kusano H, Murai K, Chiba H, Ogasawara M, Ishikawa T (1997) Three cases of branchio-oto-renal dysplasia. Otol Jpn 7:1-7

Lipkin DF, Coker NJ, Jenkins HA (1986) Hereditary congenital cholesteatoma. Arch Otolaryngol Head Neck Surg 112:1097-1100

McKusick VA (1994) Mendelian inheritance in man. Catalogs of human genes and genetic disorders, 11th edn. Johns Hopkins University Press, Baltimore

Smith RJH, Coppage KB, Ankerstjerne JKB, Capper DT, Kumar S, Kenyon J, Kimberling WJ (1992) Localization of the gene for branchiootorenal syndrome to chromosome 8q. Genomics 14:841844

Stratakis CA, Lin JP, Rennert OM (1998) Description of a large kindred with autosomal dominant inheritance of branchial arch anomalies, hearing loss, and ear pits, and exclusion of the branchiooto-renal (BOR) syndrome gene locus. Am J Med Genet 79:209-214

Vincent C, Kalatzis V, Abdelhak S, Chaib H, Compain S, Helias J, Vaneecloo FM, Petit C (1997) BOR and BO syndromes are allelic defects of EYA1. Eur J Hum Genet 5:242-246 